\title{
LETTERS
}

The purpose of this Letters section is to provide rapid dissemination of important new results in the fields regularly covered by Physics of Fluids. Results of extended research should not be presented as a series of letters in place of comprehensive articles. Letters cannot exceed three printed pages in length, including space allowed for title, figures, tables, references and an abstract limited to about 100 words. There is a three-month time limit, from date of receipt to acceptance, for processing Letter manuscripts. Authors must also submit a brief statement justifying rapid publication in the Letters section.

\section{Direct numerical simulations of three-dimensional bubbly flows}

\author{
Bernard Bunner and Grétar Tryggvason ${ }^{\text {a) }}$ \\ Department of Mechanical Engineering and Applied Mechanics, The University of Michigan, \\ Ann Arbor, Michigan 48109-2121
}

(Received 24 February 1999; accepted 23 April 1999)

\begin{abstract}
Direct numerical simulations of the motion of many buoyant bubbles are presented. The NavierStokes equation is solved by a front tracking/finite difference method that allows a fully deformable interface. The evolution of 91 nearly spherical bubbles at a void fraction of $6 \%$ is followed as the bubbles rise over 100 bubble diameters. While the individual bubble velocities fluctuate, the average motion reaches a statistical steady state with a rise Reynolds number of about 25. (C) 1999 American Institute of Physics. [S1070-6631(99)03308-5]
\end{abstract}

While direct numerical simulations, where the flow field is fully resolved and inertial and viscous effects are accounted for, have become a standard research tool in the study of the turbulent motion of homogeneous flows, such simulations are more recent for multiphase flows. The need for numerical simulations in the study of multiphase flow has been clear for some time. However, the challenge of simulating the unsteady motion of moving interfaces has led most investigators to use simplified models. For dispersed flows, where bubbles, drops, or solid particles of one phase move within another, continuous, phase, assuming Stokes flow (Charles and Pozrikidis, ${ }^{1}$ Brady, ${ }^{2}$ Loewenberg and Hinch $^{3}$ ), potential flow $\left(\right.$ Sangani ${ }^{4}$ ), or point particles (Elghobashi and Truesdell, ${ }^{5}$ Wang and Maxey, ${ }^{6}$ Squires and Eaton ${ }^{7}$ ) are typical examples of such simplifications. In this letter, results of a simulation of the motion of 91 fully deformable buoyant bubbles rising at a Reynolds number of about 25 are presented.

Esmaeeli and Tryggvason ${ }^{8,9}$ used direct numerical simulations to examine the motion of a number of rising bubbles at low, but finite, Reynolds numbers (around 1-2, depending on volume fraction and dimensionality). The simulations were done using periodic domains and included up to 324 two-dimensional bubbles or 8 three-dimensional bubbles. The simulations showed that a regular array is unstable and that it breaks up through two-bubble interactions of the "drafting, kissing, and tumbling" type (Fortes, Joseph, and Lundgren). ${ }^{10}$ Although the motion of a regular array at $O(1)$ Reynolds numbers is similar to that in Stokes flow, the evolution of the free array differs by the strong two-bubble interactions. In Esmaeeli and Tryggvason, ${ }^{11}$ the evolution was examined at a higher Reynolds number (around 20-30 for the lowest volume fraction). For the low Reynolds numbers, the freely evolving array rose faster than the regular one, in agreement with Stokes flow predictions, but at the higher Reynolds number the freely evolving array rose slower than the regular one. The effect of the number of bubbles in each periodic box was examined for the two-dimensional system, and it was found that the rise Reynolds number and the velocity fluctuations in the liquid (the Reynolds stresses) generally increase with the size of the system due to an inverse energy cascade. While some aspects of the three-dimensional flows, such as the dependency of the rise velocity on the Reynolds number, are predicted by results for twodimensional bubbles, the structure of the bubble distribution and the magnitude of the Reynolds stresses, is not. For references to other computations of bubble motions, see Esmaeeli and Tryggvason. ${ }^{9,11}$ For solid particles, Feng, Hu, and Joseph $^{12}$ have performed calculations of many particles in three dimensions. The results reported here include a much larger number of bubbles than those presented by Esmaeeli and Tryggvason and were obtained by a parallel code using the same methodology.

The motion of the bubbles is calculated by the finite difference/front tracking method developed by Unverdi and Tryggvason. ${ }^{13,14}$ The Navier-Stokes equations are solved by a second-order accurate projection method, using centered differences on a fixed, staggered grid. In order to keep the boundary between the bubbles and the ambient fluid sharp, and to accurately compute the surface tension, the phase boundary is tracked by marker points connected by triangular elements (the "front"). The maker points are advected by the fluid. As the front deforms, surface markers are dynamically added and deleted, as described in Unverdi and Tryggvason ${ }^{13}$ and Tryggvason et al. ${ }^{15}$ The surface tension is represented by a distribution of singularities (delta functions) located at the front. The gradient of the density and viscosity becomes a delta function when the change is abrupt across the boundary. To transfer the front singularities to the fixed grid, the delta functions are approximated by smooth functions with a compact support on the fixed grid. At each time step, after the front has been advected, the density and the viscosity fields are reconstructed by integration of the smooth grid-delta function. The surface tension is then added 


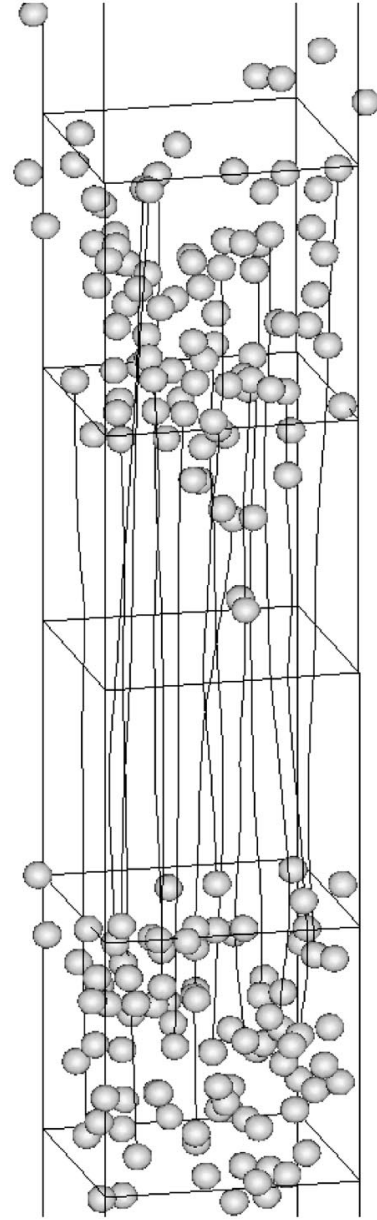

FIG. 1. The 91 bubbles at dimensionless times 32.3 and 64.4, with a few trajectories.

to the nodal values of the discrete Navier-Stokes equations. Combining the continuity equation with the momentum equation results in an elliptic equation for the pressure, which is solved by a multigrid method. This enforces a divergence-free velocity field.

The one-field approach, where the governing equations are solved simultaneously for both fluids and the interface singularities are approximated on a stationary grid, is also used in the well known volume-of-fluid (VOF) method (Scardovelli and Zaleski ${ }^{16}$ ) and in the more recent level set method. However, in these methods, the interface is located by a marker function that is advected on the fixed grid instead of by explicit marker particles as done here. For a detailed description of the method and various validation tests, see Unverdi and Tryggvason, ${ }^{13,14}$ Tryggvason et al. ${ }^{15}$ and Esmaeeli and Tryggvason. ${ }^{9}$

Accurate and fast simulations of large, well-resolved, three-dimensional bubble systems can only be obtained with parallel computing. The method was therefore reimplemented for distributed-memory parallel computers using the message passing interface (MPI) protocol. Different strategies are employed for the grid and the front. The NavierStokes solver, including the multigrid pressure solver, is parallelized by simple domain decomposition. The flow domain is partitioned into equisized subdomains, each subdomain supported by a different processor, and boundary data are

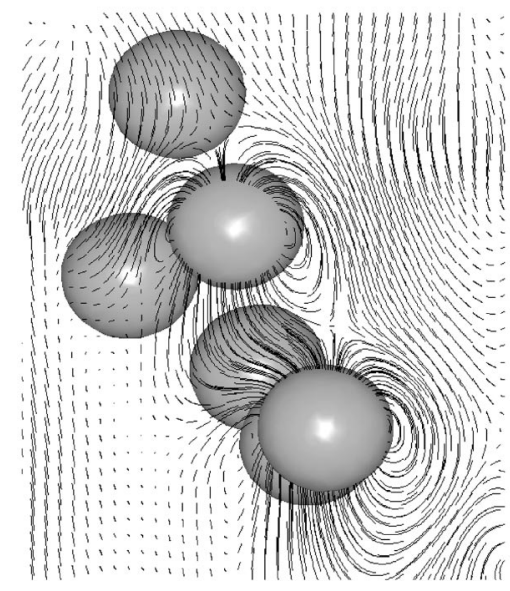

FIG. 2. Detail of the flow field at time 64.4

exchanged between adjacent subdomains. The front is parallelized by a master-slave technique which takes advantage of the nature of the physical problem to limit programming complexity and provide good performance. When a bubble is spread over more than one subdomain, one processor is designated as the "master" for that bubble. It handles and centralizes the corresponding data and sends it to the other processors, or "slaves," which then distribute the front data onto the fixed grid. While most of the code parallelizes very efficiently due to the natural load balancing of the physical problem, the parallelization efficiency is somewhat degraded by the multigrid solver. Coarse grain parallelism is therefore employed. The simulation presented here was performed on eight nodes of an IBM SP2, so that the computational domain was divided into eight subdomains. It required 30000 time steps, each step taking about $133 \mathrm{~s}$, for a total of $1100 \mathrm{~h}$ on each node.

The parallel code was used to simulate several systems with different number of bubbles in the triply periodic domain. Here, we show results from a simulation with 91 bubbles. The flow is determined by the Eötvös number, $E O$ $=\rho g d^{2} / \sigma$, the Galileo number, $N=\rho^{2} g d^{3} / \mu^{2}$ (or the Morton number, $M=g \mu^{4} / \rho \sigma^{3}$ ) and the ratios of the density and viscosity of the bubble to the ones of the ambient fluid. Here, we take $E o=1$ and $N=900\left(M=1 \cdot 23 \cdot 10^{-6}\right)$. The values of the density and viscosity inside the bubbles are chosen to be 0.02 times the values of the suspending fluid in order to make the solution of the pressure equation easier and faster. Two-dimensional tests using much smaller density ratios (Tryggvason et $a l .{ }^{15}$ ) indicate that the effect of the density ratio and of the inertia of the fluid inside the bubbles is small for these values. The domain is discretized into a $192^{3}$ grid, resulting in about 21 grid points per bubble diameter. A grid independence study has shown that this resolution leads to an accurate solution for these flow parameters.

At the initial time, the bubbles are arranged in a regular array, which is perturbed slightly by randomly moving the bubbles in every direction. When they start to rise, their wakes develop and reach their neighbors, and the array becomes unstable. Other simulations in two and three dimensions showed that the initial position of the bubbles has no influence on the results after the initial peak in the rise ve- 


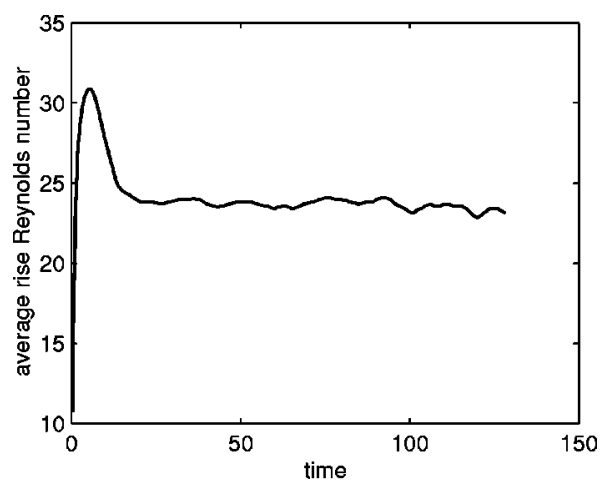

FIG. 3. Average rise Reynolds number of the bubbles.

locity. The simulations were continued until nondimensional time 128.8 , at which time the bubbles have traveled a distance corresponding to 112.3 bubble diameters or 12.6 periodic domain sizes. Time is nondimensionalized by gravity and the bubble diameter.

Figure 1 shows the bubble distribution at nondimensional times 32.2 and 64.4. The cubic boxes represent the edges of the computational domain, which are replicated by periodicity. A few trajectories are superposed on the picture. Considerable dispersion can be observed particularly in the vertical direction; at time 64.4, the bubbles are spread over three periodic domains. A detail of the flow field with a few bubbles is shown in Fig. 2, along with a few streamlines. The interaction between the bubbles can be seen in the case of the two bubbles in the front. The trailing bubble is entrained into the wake of the leading bubble. The streamlines also show a stronger recirculating flow on the trailing bubble than on the leading bubble.

The average rise Reynolds number of the bubbles is plotted in Fig. 3 versus time. The net flow rate through the boundaries of the computational domain is zero and the Reynolds number is based on the drift velocity, defined as the difference between the average velocity of the bubbles and the volume averaged velocity of the liquid. The bubbles initially reach a nearly steady rise Reynolds number corresponding to a completely regular array. As the array breaks up, the drag increases and the rise Reynolds number decreases, in agreement with the results of Esmaeeli and Tryggvason. ${ }^{11}$ Unlike both the two- and three-dimensional results of Esmaeeli and Tryggvason, the average rise velocity is essentially steady and exhibits only minor fluctuations.

The average fluctuation Reynolds number, defined as the root-mean-square value of the differences between the individual bubble velocities and their average velocity, is plotted in Fig. 4 for the vertical and horizontal directions. The fluctuations vary in time and do not settle to a constant value, even after a long time, but they appear to have reached a statistical steady state. Fluctuations in the rise direction are about three times larger than in the lateral directions.

The results presented here show the feasibility of using direct numerical simulations to examine the dynamics of finite Reynolds number bubbly flows and to provide quantitative data and insight for modeling. We believe that the present simulation will allow us to identify the dominant interaction mechanisms between the bubbles and to under-

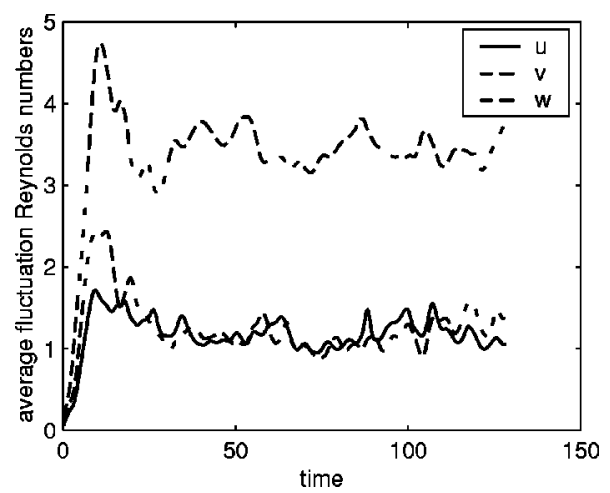

FIG. 4. Average fluctuation Reynolds number of the bubbles in the vertical and horizontal directions.

stand the microstructure of the bubble distribution. A complete analysis of these results and of the results of simulations of larger systems will be reported in a future publication.

\section{ACKNOWLEDGMENTS}

This work was supported by the National Science Foundation under Grant No. CTS-9503208. Computing services were provided by the University of Michigan Center for Parallel Computing (CPC).

${ }^{\text {a)} E l e c t r o n i c ~ m a i l: ~ g r e t a r @ e n g i n . u m i c h . e d u ~}$

${ }^{1}$ R. Charles and C. Pozrikidis, "Significance of the dispersed-phase viscosity on the simple shear flow of suspensions of two-dimensional liquid drops," J. Fluid Mech. 365, 205 (1998).

${ }^{2}$ J. F. Brady, "Stokesian dynamics simulation of particulate flow," in Particulate Two-Phase Flow, edited by M. C. Rocco (ButterworthHeinemann, Boston, 1993), pp. 912-950.

${ }^{3}$ M. Loewenberg and E. J. Hinch, "Numerical simulation of a concentrated emulsion in shear flow," J. Fluid Mech. 321, 395 (1996).

${ }^{4}$ A. S. Sangani, "Sedimentation in ordered emulsions of drops at low Reynolds number," J. Appl. Math. Phys. 38, 542 (1988).

${ }^{5}$ S. Elghobashi and G. C. Truesdell, "Direct simulation of particle dispersion in a decaying isotropic turbulence," J. Fluid Mech. 242, 655 (1992).

${ }^{6}$ L.-P. Wang and M. R. Maxey, "Settling velocity and concentration distribution of heavy particles in homogeneous isotropic turbulence,' Annu. Rev. Fluid Mech. 256, 27 (1993).

${ }^{7}$ K. D. Squires and J. K. Eaton, "Measurements of particle dispersion obtained from direct numerical simulations of isotropic turbulence," J. Fluid Mech. 226, 1 (1991).

${ }^{8}$ A. Esmaeeli and G. Tryggvason, "An inverse energy cascade in twodimensional, low Reynolds number bubbly flows," J. Fluid Mech. 314, 315 (1996).

${ }^{9}$ A. Esmaeeli and G. Tryggvason, "Direct numerical simulations of bubbly flows. Part I low Reynolds number arrays," J. Fluid Mech. 377, 313 (1998).

${ }^{10}$ A. Fortes, D. D. Joseph, and T. Lundgren, "Nonlinear mechanics of fluidization of beds of spherical particles," J. Fluid Mech. 177, 467 (1987).

${ }^{11}$ A. Esmaeeli and G. Tryggvason, "Direct numerical simulations of bubbly flows. Part II-moderate Reynolds number arrays,'” J. Fluid Mech. 385, 325 (1999).

${ }^{12}$ J. Feng, H. H. Hu, and D. D. Joseph, "Direct simulation of initial value problems for the motion of solid bodies in a Newtonian fluid, Part 1. Sedimentation,"' J. Fluid Mech. 261, 95 (1994).

${ }^{13}$ S. O. Unverdi and G. Tryggvason, "A front-tracking method for viscous, incompressible, multi-fluid flows," J. Comput. Phys. 100, 25 (1992).

${ }^{14}$ S. O. Unverdi and G. Tryggvason, "Computations of multi-fluid flows," Physica D 60, 70 (1992).

${ }^{15} \mathrm{G}$. Tryggvason, B. Bunner, O. Ebrat, and W. Tauber, "Computations of multiphase flows by a finite difference/front tracking method. I. Multifluid flows," Von Karman lecture notes, the Von Karman Institute.

${ }^{16}$ R. Scardovelli and S. Zaleski, "Direct numerical simulation of freesurface and interfacial flow,” Annu. Rev. Fluid Mech. 31, 567 (1999). 